\title{
Gastric Perforation Because of Non-Invasive Positive-Pressure Ventilation: Review of Complications
}

\author{
Takeshi Nishimura, Kunihoro Shirai,, Atsunori Nakao, ${ }^{2}$ and Joji Kotani ${ }^{1}$
}

\begin{abstract} pediatrics on post-admission day 26.

$\mathbf{N}^{\circ}$ ON-INVASIVE POSITIVE-PRESSURE VENTILATION (NIPPV) is used widely on patients with chronic respiratory failure due to neuromuscular disease. NIPPV is a safe, portable, and effective procedure that can circumvent potential complications associated with invasive mechanical ventilation, such as nosocomial pneumonia and tracheal trauma. Because NIPPV can be used intermittently, ventilation does not interfere with eating and speaking.

The most frequent problem that might occur is development of an ulcer where the mask makes contact with the face. However, some life-threatening complications associated with NIPPV have been documented. We experienced a case of gastric perforation presumably due to high respiratory pressure support and reviewed the published complications following NIPPV.
\end{abstract}

Background: Non-invasive positive-pressure ventilation (NIPPV) is a useful tool, especially for patients with respiratory failure. Although NIPPV is accepted now as harmless and comfortable treatment tools for patients, the associated equipment can induce a critical illness if expiratory pressure is not adjusted adequately.

Case: A 23-year-old male was admitted to our hospital complaining of severe abdominal pain. He had a history of congenital hypomyelination neuropathy. Because of respiratory insufficiency due to neuropathy, his doctor had prescribed NIPPV and increased end expiratory pressure three months earlier. Because abdominal computed tomography (CT) revealed ascites and pneumoperitoneum indicating gastrointestinal perforation, emergent laparotomy was performed. Intra-operative findings showed a gastric perforation and hematoma at the gastric anterior walls. The patient recovered from post-operative bowel obstruction and was transferred to

Conclusion: Although gastrointestinal complications associated with NIPPV are rare, clinicians should be aware of possible life-threatening adverse events of the gastrointestinal rupture due to high respiratory pressure support.

\section{Case Report}

A 23-year-old male was transferred to our department complaining of severe abdominal pain. He had a history of a hump back, muscle atrophy, scoliosis, and congenital hypomyelination neuropathy. No intellectual disability was identified. His height was $90 \mathrm{~cm}$ and his body weight was $20 \mathrm{~kg}$. The patient required NIPPV to support respiratory failure caused by neuropathy. No medications, including steroids, had been prescribed recently for him. His physical findings revealed a hard and extended abdomen and rebound tenderness. His laboratory test results on arrival were as follows: White blood cell count, 3020/ $\mu \mathrm{l}$; hemoglobin, $18.9 \mathrm{~g} / \mathrm{dl}$, platelet count, $31.3 \times 10^{4} / \mu \mathrm{l}$; C-reactive protein, $9.44 \mathrm{mg} / \mathrm{dl}$; albumin, $4.9 \mathrm{~g} / \mathrm{dl}$; total bilirubin, $1.0 \mathrm{~g} / \mathrm{dl}$; D-dimer, $1.6 \mu \mathrm{g} / \mathrm{ml}$; prothrombin time, 15.9 seconds.

Chest radiograph and computed tomography (CT) showed pneumoperitoneum and intra-abdominal fluid collection around the liver (Fig. 1). Emergent laparotomy was performed because we suspected upper intestinal perforation. Surgical findings demonstrated gastric perforation and multiple hematomas of the intra-stomach walls (Fig. 2). Surgical repair, resection of necrotic gastric walls, and establishment of a jejunostomy prevented exacerbation of his condition.

\footnotetext{
${ }^{1}$ Department of Emergency, Disaster, and Critical Care Medicine, Hyogo College of Medicine, Nishinomiya City, Hyogo, Japan.

${ }^{2}$ Department of Emergency and Critical Care Medicine, Okayama University, Graduate School of Medicine, Dentistry, and Pharamceutical Sciences, Okayama, Japan.

(C) Takeshi Nishimura et al. 2016; Published by Mary Ann Liebert, Inc. This Open Access article is distributed under the terms of the Creative Commons License (http://creativecommons.org/licenses/by/4.0), which permits unrestricted use, distribution, and reproduction in any medium, provided the original work is properly credited.
} 


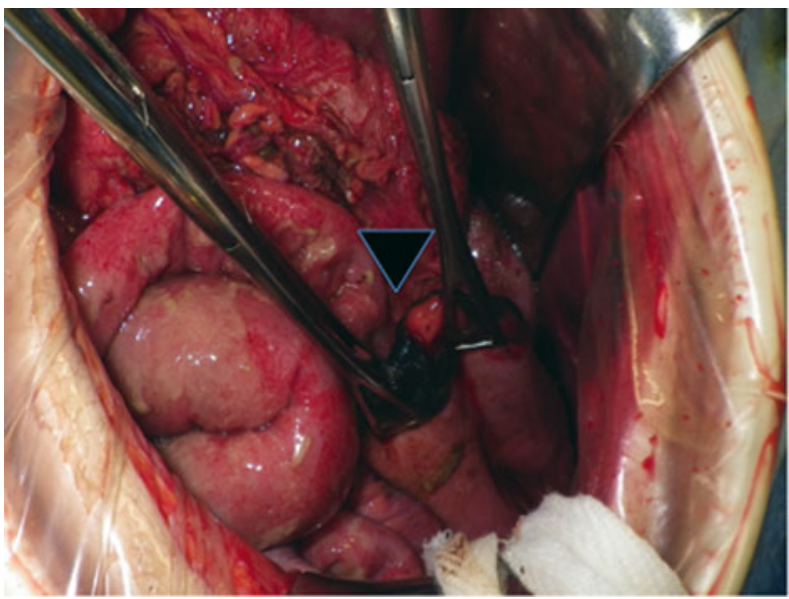

FIG. 1. Arrow demonstrates perforation site and hematoma at gastric anterior wall.

Histopathological examination of the surgical specimens showed hemorrhage and transmural necrosis in the stomach wall. No malignant cells, abnormal vessels, or nerve plexus were identified.

On post-admission day 12 , the patient complained of nausea and abdominal pain. Computed tomography revealed mechanical bowel obstruction around the jejunostomy tube and intestinal pneumatosis. Suspecting strangulation of the ileus, emergent laparotomy was performed. Intra-operative findings disclosed an obstruction of the upper intestine at a site in the left upper quadrant between the jejunostomy tube and rib cage. Release of the strangulation without intestinal resection and removal of the jejunostomy improved his condition. No events occurred post-operatively and the patient was transferred to the pediatrics department 26 days later.

We assumed that overwhelming expiratory pressure with NIPPV might cause a stomach distention; therefore, multiple hematomas in the stomach wall occurred, followed by worsening of the hematoma and leading to gastric perforation and pneumoperitoneum. The patient has been well on mechanical ventilation via a tracheostomy enabling comfortable respiration without NIPPV.

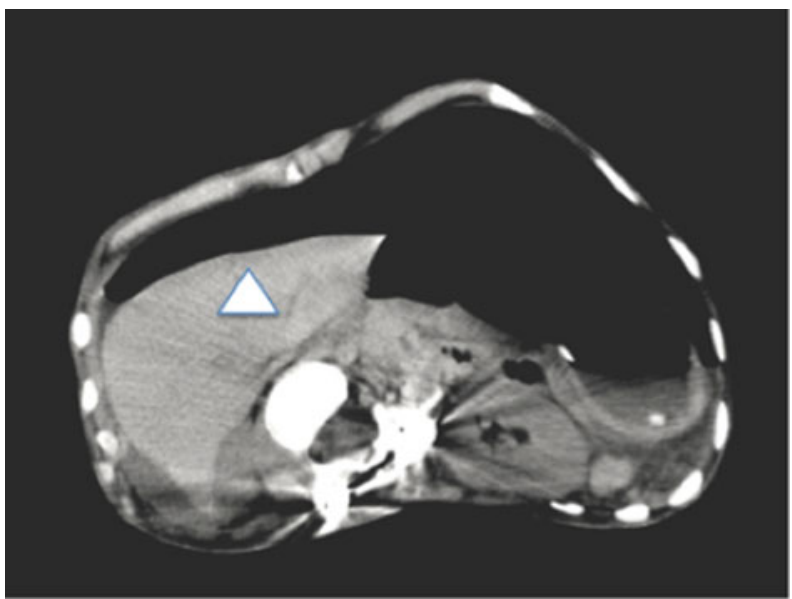

FIG. 2. Arrow head demonstrates ascites and pneumoperitoneum around liver, which indicates upper intestinal perforation.

\section{Discussion}

Non-invasive positive-pressure ventilation has gained wide acceptance as an effective treatment for the management of patients with respiratory failure. Non-invasive positive-pressure ventilation is considered safe because it can prevent potential complications associated with invasive mechanical ventilation such as nosocomial pneumonia and tracheal barotrauma, which may occur with invasive mechanical ventilation [1]. Non-invasive positive-pressure ventilation contributes to the reduction of mortality, morbidity, and shortness of hospital length of stay for patients with respiratory failure [2]. Clinicians should be aware that the efficacy of NIPPV, as well as contraindications, include consciousness disorders or unstable hemodynamic state [3].

Although NIPPV is a fairly safe method used as the firstline ventilator support in patients with respiratory failure, Carron et al. reported the complications of NIPPV in a qualitative review of randomized trials [4]. According to these trials, aspiration pneumonia, barotrauma, and hypotension may occur in less than $5 \%$ of patients, rarely, and infrequently, respectively. Associated with problems related to interface-ventilator interactions during NIPPV and remedies, various complications such as arm edema, $\mathrm{CO}_{2}$ rebreathing, claustrophobia, discomfort, nasal and skin abrasion, noise, and patient-ventilator dyssynchrony can occur. Moreover, aerophagia is common. Airway dryness, facial skin erythema, nasal congestion, nose/sinus/ear pain, gastric insufflation, and orthodontic problems have arisen in less than $50 \%$ cases associated with air pressure and flow. Lechtzin et al. reported that a patient with amyotrophic lateral sclerosis in whom NIPPV had already been indicated died due to NIPPV equipment failure. They emphasized that patients and their caregivers should be counseled that NIPPV is not a simple substitute for tracheostomy and mechanical ventilation and do not hesitate to indicate tracheostomy and mechanical ventilation if needed [5].

Why gastric perforation occurred in our patient is unclear. Positive-pressure ventilation without tracheal intubation may cause greater cricopharyngeal pressures and may lead to an increase in intragastric pressure and dilatation of the stomach. Although congenital hypomyelination neuropathy might affect this condition, surgical specimens did not show an atrophy of vessels or nerve plexus in the gastric wall. On the basis of the history of an increase of NIPPV pressure and frequent insufflation and aerophagia, it may be reasonable that NIPPV-induced excessive pressure on the stomach leads to perforation.

In rare instances, upper gastrointestinal perforation is a documented complication of nasopharyngeal oxygen administration. Fifteen cases of neonatal gastrointestinal perforations not associated with necrotizing entercolitis or bowel obstruction at Milwaukee Children's Hospital were reviewed [6]. All the children with gastrointestinal perforation were ventilated by facemask or nasal prongs at the time of rupture. The review demonstrated that facemask- or nasal-prongcycled ventilation in infants was associated with an almost 30-fold increase in the risk of developing a gastric or intestinal perforation when compared with infants ventilated with endotracheal tubes.

Four previous case reports associated with intestinal perforation induced by NIPPV hyperinflation were found [7-10]. 
Two cases of Boerhaave syndrome, esophageal pneumatosis, and gastric perforation were published. Although two cases might have survived, two patients with Boerhaave syndrome died with severe systemic inflammatory response and multiple organ failure. To our knowledge, our patient is the second reported adult case of the gastric perforation receiving NIPPV treatment.

\section{Conclusion}

Although NIPPV is performed with better equipment for patients with respiratory failure, some complications, including not only minor diseases such as erythema, ulceration, and mouth dryness, but also severe diseases such as gastric insufflation leading to perforation, might occur. As emergency clinicians, we should be aware of these complications. NIPPV is not always conducted with non-invasive equipment. Acknowledgment and recognition of the prevention of and complications associated with NIPPV can lead to appropriate use of the treatment, depending on the patient's condition.

\section{Author Disclosure Statement}

The authors declare no conflicts of interest.

\section{References}

1. Brochard L. Mechanical ventilation: Invasive versus noninvasive. Eur Respir J Suppl 2003;47:31s-37s.

2. Brochard L, Mancebo J, Wysocki M. Noninvasive ventilation for acute exacerbation of chronic obstructive pulmonary disease. N Engl J Med 1995;333: 817-822.

3. Brochard L. Mechanical ventilation: Invasive versus noninvasive. Eur Respi J 2003;22:Suppl47:31s-37s.

4. Carron M, Freo U, Bahammam AS. Complications of noninvasive ventilation techniques: A comprehensive qualitative review of randomized trials. Brit J Anesth 2013;5:1-19.

5. Lechtzin N, Weiner CM, Clawson L. A fetal complication of noninvasive ventilation. N Engl J Med 2001;344:7.

6. Jeffery SG, David BN, Thomas R. Increased risk of gastrointestinal perforations in neonates mechanically venti- lated with either face mask or nasal prongs. Pediatrics 1985;76:3.

7. Bakkali H, Aissa I, Massou S: Unusual complication of noninvasive ventilation: The oesogastric pneumatosis associated with a subcutaneous emphysema. Rev Pneumol Clin 2014:70:236-239.

8. Van de Louw A, Broccs E, Boiteau R. Oesphageal perforation associated with noninvasive ventilation: A case report. Chest 2002;122:1857-1858.

9. Jean-Lavaleur M, Perrier V, Roze H: Stomach rupture associated with noninvasive ventilation. Annales Francaises d'Anesthesie et de Reanimation 2009;28:588-591.

10. Patel MS, Chakraborty PU. A case of Boerhaave's syndrome presenting after a trial of non-invasive ventilation Med Princ Pract 2009;18:155-158.

Address correspondence to:

Dr. Atsunori Nakao

Department of Emergency and Critical Care Medicine

Okayama University

Graduate School of Medicine, Dentistry, and Pharmaceutical Sciences 2-5-1 Shikata-cho, Kita-ku Okayama, 700-8558, Japan

E-mail: atsunorinakao@aol.com

$$
\begin{aligned}
\text { Abbreviations Used } & \\
\mathrm{CT} & =\text { computed tomography } \\
\mathrm{NIPPV} & =\text { non-invasive positive-pressure ventilation }
\end{aligned}
$$

Cite this article as: Nishimura T, Shirai K, Nakao A, and Kotani J (2016) Gastric perforation due to non-invasive positive-pressure ventilation: Review of complications, Surgical Infections Case Reports 1:1, 41-43, DOI: 10.1089/crsi.2016.29008.tn 